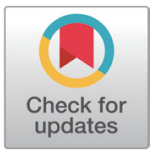

Received: Nov 26, 2020

Revised: Feb 8, 2021

Accepted: Feb 18, 2021

\#These authors contributed equally to this work.

${ }^{*}$ Corresponding author Jee Soo Son

iNtRON Biotechnology, Seongnam

13202, Korea.

Tel: +82-31-739-5309

E-mail: jsson@intron.co.kr

Ji Hyung Kim

Infectious Disease Research Center

Korea Research Institute of Bioscience and Biotechnology, Daejeon 34141,

Korea.

Tel: +82-42-879-8272

E-mail: kzh81@kribb.re.kr

Copyright ( $\odot 2021$ Korean Society of Animal Sciences and Technology. This is an Open Access article distributed under the terms of the Creative Commons Attribution Non-Commercial License (http:// creativecommons.org/licenses/bync/4.0/) which permits unrestricted non-commercial use, distribution, and reproduction in any medium, provided the original work is properly cited.

ORCID

Seon Young Park

https://orcid.org/0000-0002-1351-7557 In Hwang Kim

https://orcid.org/0000-0002-0141-5537

\section{Complete genome sequence of serotype 3 Streptococcus suis INT- 01 , isolated from a domestic pig in Korea}

\author{
Seon Young Park ${ }^{1,2 \#}$, In Hwang Kim" ${ }^{3 \#}$, Hyun Jin Yu ${ }^{3}$, Hyoung Rok Paik ${ }^{3}$, \\ Jee Soo Son ${ }^{3 *}$ and Ji Hyung Kim ${ }^{1 *}$ \\ ${ }^{1}$ Infectious Disease Research Center, Korea Research Institute of Bioscience and Biotechnology, \\ Daejeon 34141, Korea \\ ${ }^{2}$ Division of Animal and Dairy Sciences, College of Agriculture and Life Science, Chungnam National \\ University, Daejeon 34134, Korea \\ ${ }^{3}$ iNtRON Biotechnology, Seongnam 13202, Korea
}

\begin{abstract}
Streptococcus suis is a major pig pathogen causing severe economic losses to the swine industry. This study aimed to analyze the genome of $S$. suis strain INT-01 isolated from a domestic pig in Korea. We found that the genome of strain INT-01 contains 2,092,054 bp, with a guanine $(\mathrm{G})+$ cytosine $(\mathrm{C})$ content of $41.3 \%$, and the capsular polysaccharide synthesis locus of this strain is almost identical to that of serotype $3 \mathrm{~S}$. suis strain 4961 isolated from China, suggesting that these isolates can be classified as serotype 3. Genomic analyses revealed that strain INT-01 is an extracellular protein factor $(e p f)^{-} /$muraminidase-released protein $(m r p)^{+} /$suilysin $(s / y)^{-}$S. suis, which is the most prevalent genotype in Korea, and several virulence-related genes associated with the pathogenicity of $S$. suis were also detected. The genomic information of strain INT-01 may provide important insights into the development of control strategies against $S$. suis infections in Korea.
\end{abstract}

Keywords: Streptococcus suis, Pathogen, Genotype, Control strategy, Swine industry

Streptococcus suis is a major pig pathogen causing severe economic losses to the swine industry and is considered a prominent zoonotic agent [1]. Thus far, 35 capsular serotypes have been reported for this species, and its distribution among pigs geographically varies [2]. Several virulence factors have been reported for S. suis, and extracellular protein factor (epf), muraminidase-released protein ( $m r p)$, and suilysin $(s l y)$ are considered to be the major virulence-associated genes underlying the pathogenesis of this bacterium [3]. Although a recent study has reported that serotype 3, with the epf/mrp $p^{+} / s^{-}$ genotype, is the most prevalent serotype among Korean isolates [1], its genomic characteristics remain unknown.

In this study, strain INT-01 was isolated in 2018 from the tonsillar swab of a growing pig with respiratory clinical signs that had been reared at a private farm in Yesan (Chungcheongnam-do, Korea). The $\alpha$-hemolytic isolate displayed 99.7\% 16S rRNA identity to S. suis $\mathrm{S} 735^{\mathrm{T}}$ (AY585196), thus suggesting that strain INT-01 is a member of $S$. suis. The antimicrobial susceptibility of strain INT- 
Hyun Jin Yu

https://orcid.org/0000-0001-9930-0318

Hyoung Rok Paik

https://orcid.org/0000-0002-6343-2383

Jee Soo Son

https://orcid.org/0000-0003-0094-2105

Ji Hyung Kim

https://orcid.org/0000-0002-7921-2625

Competing interests

No potential conflict of interest relevant to

this article was reported.

Funding sources

This study was funded by the KRIBB Initiative programs, the National Research Foundation (NRF) of Korea (NRF-2020R1I1A2068827), the Collaborative Genome Program of the Korea Institute of Marine Science and Technology Promotion (20180430) funded by the Ministry of Oceans and Fisheries, and ATC Program grant 10076996 from the Ministry of Trade, Industry, and Energy of Korea.

Acknowledgements

Not applicable.

Availability of data and material Upon reasonable request, the datasets of this study can be available from the corresponding author. Streptococcus suis INT-01 has been deposited in the Culture Collection of Antimicrobial Resistant Microbes (CCARM) as CCARM4634

Authors' contributions

Conceptualization: Son JS, Kim JH Data curation: Park SY, Kim IH, Yu HJ. Formal analysis: Park SY, Kim IH. Methodology: Park SY, Kim IH, Yu HJ, Paik HR.

Software: Park SY, Kim IH

Validation: Park SY, Kim IH, Paik HR.

Investigation: Park SY, Kim IH, Kim JH.

Writing - original draft: Park SY, Kim IH, Kim $\mathrm{JH}$

Writing - review \& editing: Son JS, Kim JH.

Ethics approval and consent to participate This article does not require IRB/IACUC approval because there are no human or animal participants.
01 was evaluated according to the testing guidelines and interpretive breakpoints in the M100S document of the Clinical and Laboratory Standards Institute [4]. The isolate was resistant to tetracycline, erythromycin, gentamicin, lincomycin, and levofloxacin. Its genome was sequenced using the PacBio RS II system (Pacific Biosciences, Menlo Park, CA, USA) with P6-C4 chemistry by constructing a $20 \mathrm{~kb}$ SMRTbellTM template library, using the DNA/polymerase binding kit P6 (Pacific Biosciences) in accordance with the manufacturer's instructions. Genome assembly of the filtered reads $\left(1,245,264,540 \mathrm{bp}, 153,790\right.$ reads, $\left.N_{50}, 10,696 \mathrm{bp}\right)$ was performed using the PacBio HGAP (v.2.3) pipeline with default settings, using the de novo assembly protocol, and the reads (2,113,334 bp, 409× were polished using Quiver. Annotation was performed using the Prokaryotic Genome Annotation Pipeline (National Center for Biotechnology Information, http://www.ncbi. nlm.nih.gov/books/NBK174280/).

The genome of INT-01 was found to comprise 2,092,054 bp (41.3\% guanine [G] + cytosine [C] content), encoding 2,054 coding sequences, 12 rRNAs, 57 tRNAs, and 4 non-coding RNAs (Table 1). Genome similarities among INT-01 and other S. suis strains were assessed using ANI Calculator [5], and the genome was found to be $96.9 \%$ similar to that of S. suis $\mathrm{S} 735^{\mathrm{T}}$ (NC_018526, serotype 2); furthermore, this strain is most similar to S. suis ST3 (NC_015433, serotype 3) [6,7] (Fig. 1). Moreover, the capsular polysaccharide synthesis (cps) locus of INT-01 is almost identical (> 99.9\%) to that of strain 4961 (JF273646, serotype 3), thus suggesting that INT-01 can be classified as serotype 3 [8].

Major virulence-associated genes in INT-01 were manually compared with those of the $S$. suis strains available in the GenBank database, and other virulence-associated and antibiotic-resistant genes were screened as previously described [9]. Consequently, strain INT-01 was identified to be an epf/mrp ${ }^{+} / s l y-S$. suis, which is the most prevalent genotype in Korea [1]. Additionally, several virulence-associated factors of the genus Streptococcus, including choline-binding protein D $(\operatorname{cbp} D)$, fibronectin/fibrinogen-binding proteins ( $f b p 54 / p a v A)$, glyceraldehyde-3-phosphate dehydrogenase $(p l r / g a p A)$, extracellular hyaluronidase $(b y l A)$, periplasmic serine endoprotease $(b \operatorname{tr} A / \operatorname{deg} P)$, trigger factor $(\operatorname{tig} / r o p A)$, and zinc metalloproteinase $(z m p C)$ were detected. However, no genetic determinants associated with antibiotic resistance were detected. These results suggest that other unknown resistance determinants may present in INT-01, warranting further investigation. To our knowledge, this is the first study to report the genome of S. suis from Korea. The present results potentially provide important insights into the development of control strategies against $S$. suis infections by providing the genomic information of the most prevalent type of the pig pathogen in Korea.

Streptococcus suis INT-01 has been deposited in the Culture Collection of Antimicrobial

Table 1. Characteristics of the Streptococcus suis strain INT-01 genome

\begin{tabular}{|c|c|}
\hline Features & Value \\
\hline Genome size (bp) & $2,092,054$ \\
\hline $\mathrm{G}+\mathrm{C}$ content $(\%)$ & 41.3 \\
\hline Contigs & 1 \\
\hline Total genes & 2,073 \\
\hline tRNAs & 58 \\
\hline rRNAs (5S, 16S, 23S) & $4,4,4$ \\
\hline ncRNAs & 4 \\
\hline Protein-coding genes & 1,929 \\
\hline Pseudogenes & 70 \\
\hline
\end{tabular}




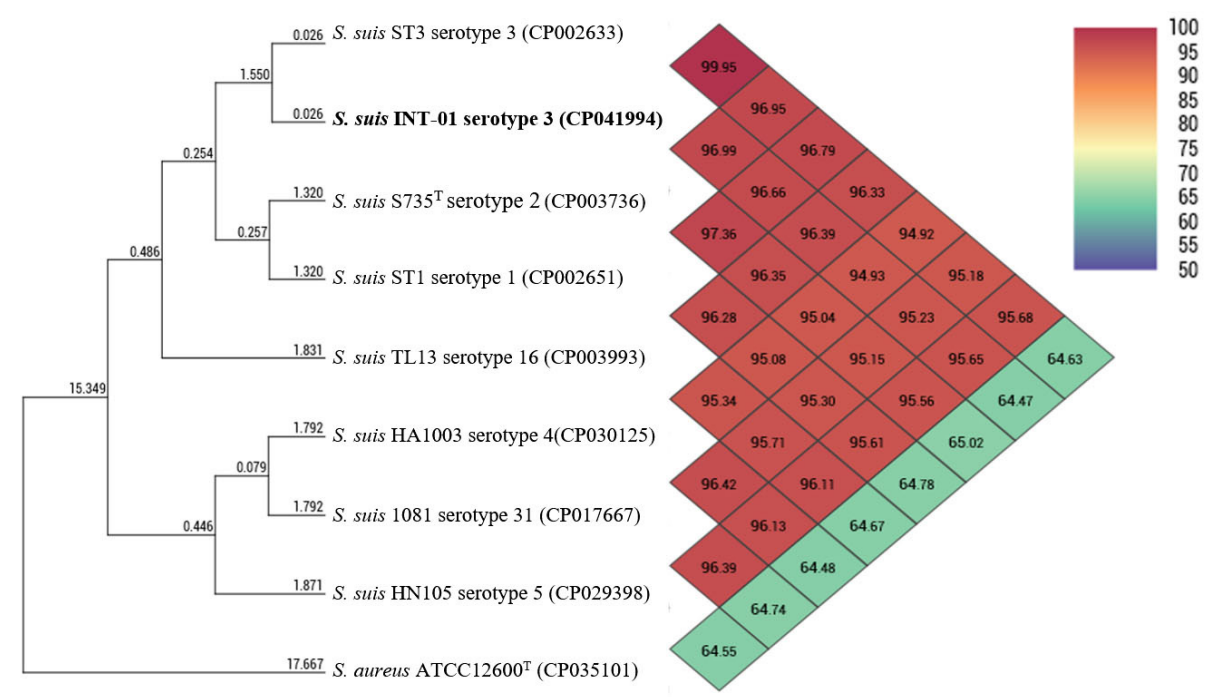

Fig. 1. Overall genome relatedness and heatmaps on the basis of OrthoANI values determined with the available complete genomes of Streptococcus suis INT-01 and other species in the S. suis strains. The result of each two-strain comparison is provided, where the diagonals departing from each strain meet, e.g., the OrthoANI value between S. suis INT-01 and S. suis ST3 is 99.95\% (2-column fitting image).

Resistant Microbes (CCARM) as CCARM4634, and its genome has been deposited in the DDBJ/ENA/GenBank database under accession number CP041994.1.

\section{REFERENCES}

1. Oh SI, Jeon AB, Jung BY, Byun JW, Gottschalk M, Kim A, et al. Capsular serotypes, virulence-associated genes and antimicrobial susceptibility of Streptococcus suis isolates from pigs in Korea.J Vet Med Sci. 2017;79:780-7. https://doi.org/10.1292/jvms.16-0514

2. Athey TBT, Teatero S, Lacouture S, Takamatsu D, Gottschalk M, Fittipaldi N. Determining Streptococcus suis serotype from short-read whole-genome sequencing data. BMC Microbiol. 2016;16:162. https://doi.org/10.1186/s12866-016-0782-8

3. Li LL, Liao XP, Sun J, Yang YR, Liu BT, Yang SS, et al. Antimicrobial resistance, serotypes, and virulence factors of Streptococcus suis isolates from diseased pigs. Foodborne Pathog Dis. 2012;9:583-8. https://doi.org/10.1089/fpd.2011.1106

4. Clinical and Laboratory Standards Institute [CLSI]. performance standards for antimicrobial susceptibility testing M100S. 29th ed. Wayne, PA: CLSI; 2018.

5. Lee I, Ouk Kim Y, Park SC, Chun J. OrthoANI: an improved algorithm and software for calculating average nucleotide identity. Int J Syst Evol Microbiol. 2016;66:1100-3. https://doi. org/10.1099/ijsem.0.000760

6. Hu P, Yang M, Zhang A, Wu J, Chen B, Hua Y, et al. Complete genome sequence of Streptococcus suis serotype 3 strain ST3. J Bacteriol. 2011;193:3428-9. https://doi.org/10.1128/ JB.05018-11

7. Goyette-Desjardins G, Auger JP, Xu J, Segura M, Gottschalk M. Streptococcus suis, an important pig pathogen and emerging zoonotic agent—an update on the worldwide distribution based on serotyping and sequence typing. Emerg Microbes Infect. 2014;3:1-20. https://doi. org/10.1038/emi.2014.45

8. Wang K, Fan W, Cai L, Huang B, Lu C. Genetic analysis of the capsular polysaccharide 
synthesis locus in 15 Streptococcus suis serotypes. FEMS Microbiol Lett. 2011;324:117-24. https://doi.org/10.1111/j.1574-6968.2011.02394.x

9. Lee K, Kim HK, Sohn H, Cho Y, Choi YM, Jeong DG, et al. Genomic insights into Photobacterium damselae subsp. damselae strain $\mathrm{KC}-\mathrm{Na}-1$, isolated from the finless porpoise (Neophocaena asiaeorientalis). Mar Genom. 2018;37:26-30. https://doi.org/10.1016/j.margen.2017.09.004 